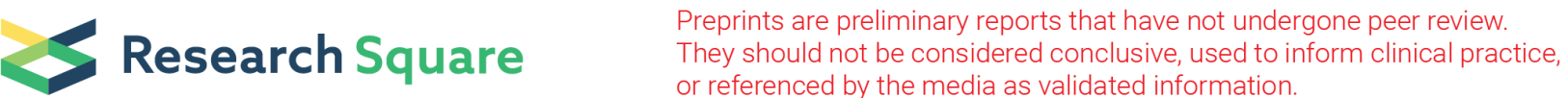

\section{Inflammatory Cytokine Neutralizing Antibody Treatment Could Recover Increased Follicular Helper and Follicular Regulatory T Cells in Murine Models of Arthritis}

\author{
Xingyue Zeng \\ Peking University People's Hospital \\ Meng Li \\ Peking University People's Hospital \\ Mohan Zheng \\ Peking University Health Science Center \\ Tianci Liu \\ Peking University People's Hospital \\ Rui Kang \\ Peking University People's Hospital \\ Lijuan Xu
}

Peking University Health Science Center

Qinzhu Xu

Peking University People's Hospital

\section{Songsong Lu}

Peking University People's Hospital

\section{Ying Song}

Peking University People's Hospital

Chen Liu ( $\boldsymbol{V}$ liuchen-best@pku.edu.cn )

Peking University People's Hospital https://orcid.org/0000-0001-7120-5626

\section{Research Article}

Keywords: TFH, TFR, collagen-induced arthritis, rheumatoid arthritis, inflammatory cytokines

Posted Date: September 17th, 2021

DOl: https://doi.org/10.21203/rs.3.rs-880040/v1

License: (c) (i) This work is licensed under a Creative Commons Attribution 4.0 International License. Read Full License 
Version of Record: A version of this preprint was published at Journal of Inflammation Research on July 14th, 2022. See the published version at https://doi.org/10.2147/JIR.S355720. 


\section{Abstract}

Objective. We aimed to illustrate the changes of follicular helper (TFH) and follicular regulatory (TFR) cells in rheumatoid arthritis using the collagen-induced arthritis (CIA) model and clarity the impact of antiinflammatory treatment on TFH and TFR cells.

Methods. We established 10-week-old CIA model and used flow cytometry to analyze the changes of TFH and TFR cells in peripheral blood and spleen at different time points ( $5 w, 7 w, 10 w, 13 w)$. The expression of TIGIT, CD226, ICOS and PD-1 characterizing the functions of TFH and TFR were also analyzed. The function of spleen TFH and TFR cells from CIA was further analyzed. The effects of anti-inflammatory antibody treatments on the subpopulation and function changes of TFH and TFR were also analyzed in CIA mice.

Results. The levels of TFH and TFR were significantly increased in spleen and peripheral blood of CIA mice. After treatment, TFH and TFR cells decreased significantly. TIGIT+ and TIGIT+CD226-TFH cells in CIA mouse spleen were elevated and PD-1 and ICOS expression on TFH and TFR cells in the spleen were also significantly increased. The ability of TFH to secrete IL-21 and help B cells, and TFR to secrete IL-10 and inhibit TFH were both enhanced in CIA mouse spleen. After antibody treatment, cell subsets and functions were significantly recovered.

Conclusion. In the pathogenesis of rheumatoid arthritis, TFH and TFR cells in the germinal center increases and their functions are enhanced. After the treatment by inflammatory factor antibodies, TFH and TFR subsets and their functions can be significantly recovered.

\section{Highlights}

1. TFH and TFR were significantly increased in spleen and peripheral blood of CIA mice.

2. PD-1 and ICOS expressions on TFH and TFR cells in the spleen of CIA mice were up-regulated.

3. The ability of TFH and TFR cells were both enhanced in CIA mice.

4. After inflammatory factor neutralizing antibody treatment, TFH and TFR functions were recovered.

\section{Introduction}

Rheumatoid Arthritis (RA) is a clinically common chronic autoimmune disease with symmetry peripheral multi-joint disease, and its main pathology is shown as inflammatory cell infiltration, the formation of angiogenesis, joint cartilage destruction, and further leads to joint malformations or even function loss[1, 2]. One of the features of RA is to produce a variety of autoantibodies [3], and it is currently believed that pathogenic autoantibodies caused by abnormal $T$ and $B$ lymphocyte functions are the important factor in the pathogenesis of RA[4]. However, the detailed pathogenesis of rheumatoid arthritis so far is not fully clarified, and the relevant regulation mechanism remains unclear. 
The CD4 + T cells play very important roles in the pathogenesis of rheumatoid arthritis. Studies have shown that $\mathrm{TH} 17$ cells with pro-inflammatory effects and regulatory T cells (Treg) are with opposite roles to autoimmune and inflammatory responses [5-7]. In recent years, more and more studies have shown that the specific CD $4+T$ cell subset- follicular helper T cells (TFH) play a key role in humoral immunity[810].TFH cells are important for germinal center formation and humoral responses, which can help $B$ cells by supporting class switching, somatic hypermutation, and high affinity autobody production[8-10]. The role of TFH cells in autoimmune diseases has received more and more attention[4, 11-15]. TFH cells rise in immune disease, and promoted immune disease [4, 11-15]. The increase in peripheral blood TFH-like cells was detected in patients with primary Sjogren's syndrome, dermatomyositis and systemic lupus erythematosus (SLE) and produced extensive autoantibodies [11-13]. In terms of rheumatoid arthritis, circulating CD4 + CXCR5+, CD4 + CXCR5 + PD1 + and CD4 + CXCR5 + PD1hi TFH cells were reported to be increased $[4,14,15]$.

TFH cell-mediated humoral immunity is suppressed by follicular regulatory T cells (TFR). TFR is a specific subset of Treg cells expressing CXCR5, which has been widely concerned and studies in recent years[16-21]. TFR cells express FoxP3, Bcl-6 and BliMP-1 transcription factors and are with the function of inhibiting TFH cells and germinal center responses[16]. Animal experiments show that the lack of TFR cells can lead to a larger germinal center reaction in vivo[17], TFR condition-knockout can promote autoimmune disease in mice[18]. At present, research on the role of TFR in autoimmune diseases is still on the way. Compared with the health control, the frequency of CD4 + CXCR5 + FOXP3 + TFR cells in peripheral blood of ankylosing spondylitis (AS) patients was significantly increased[19]. Our group found that the number of CD4 + CXCR5 + FOXP3 + TFR cells in the peripheral blood of SLE patients was significantly increased compared to health control[20]. As for the role of TFR in RA, our research showed that the peripheral blood CXCR5 + FoxP3 + TFR cell level and the TFR/TFH ratio in the peripheral blood of patients with stable remission RA were significantly increased compared with active active RA patients and healthy controls[21]. In addition, TFR cells were negatively correlated with DAS28 scores that reflect the condition of RA, suggesting that TFR cells in RA patients have a negative correlation with RA[21].

The collagen-induced arthritis (CIA) model is one of the most studied autoimmune models of rheumatoid arthritis. CIA model has been widely used to identify potential pathogenic mechanisms of autoimmunity in RA, including the role of various types of cells in the pathogenesis and progression of RA disease [22, 23]. CIA model also plays an important role in the testing and development of new biology-based treatments for autoimmune diseases $[22,23]$. In order to clarify the role of TFH and TFR in depth in RA, we used CIA model to explore the changes and functions of TFR and TFH in germinal centers.

In addition, the changes in the expression and function of TFH and TFR during the treatment of RA have also attracted our attention. One of the treatments for RA is to use neutralizing antibodies against inflammatory cytokines. Clinically, neutralizing antibody drugs related to inflammatory factors have been used in the treatment of RA, including infliximab (monoclonal anti-TNFa antibody) and canakinumab (monoclonal anti-IL-1 $\beta$ antibody)[24-27].The inflammatory cytokine TNF leads to the pathogenesis of various rheumatic diseases and is produced by cells of the innate and adaptive immune system[24]. RA 
patients often have high TNF levels in synovial fluid and synovial tissues, and TNF inhibitors have been shown to be effective in the treatment of RA $[24,25]$. It has been proven that the neutralizing anti-IL-1 $\beta$ monoclonal antibody canakinumab has significant efficacy in juvenile RA [26]. In patients with active RA, adding canakinumab to MTX treatment can improve the treatment effect [27]. Although these antibodies are used to treat RA, how they affect TFH and TFR has not been elucidated.

In this study, we used the CIA mouse model to deeply study the expression and function changes of TFH and TFR in the germinal center of the spleen, and on this basis, we explored the effect of inflammatory factor neutralizing antibody treatment on the expression and function of TFH and TFR in vivo. Our research will help scientists and clinicians deepen their understanding of the role of TFH and TFR in the pathogenesis and treatment of RA.

\section{Materials And Methods}

\subsection{Mice}

Studies were conducted by use of 10-week-old female DBA/1 mice, purchased from Charles River (Charles River Laboratory Animal, Beijing, China). All mice received human care and the research protocol complied with the Animal Research: Reporting of in Vivo Experiments (ARRIVE) guidelines, with approval from ethical board of Peking University People's Hospital.

\subsection{Induction and assessment of CIA}

Bovine type II collagen (Chondrex, Redmond, WA, USA) was emulsified in Freund's complete adjuvant (FCA; Sigma-Aldrich, St. Louis, MO) with equal volume. DBA/1 mice were intradermally (i.d.) immunized at tail base with $100 \mu \mathrm{l}$ of emulsion $(150 \mu \mathrm{g}$ of bovine $\mathrm{CII})$. After 21 days, mice received a second intradermal immunization with $150 \mu \mathrm{g}$ Cll emulsified in Freund's incomplete adjuvant (FIA; Sigma-Aldrich, St. Louis, MO). CIA severity was determined by assessment of overall inflammation in individual paws and each paw was graded according to the following: 0 No inflammation, 1 swelling of at least 1 finger, 2 swelling of each finger and paw inflammation, 3 severe inflammation or rigidity of the entire paw and finger, 4 necrosis. The total clinical score is the sum of these 4 scores, and the maximum clinical score of each mouse is 16 . In terms of treatment, starting from the $8 \mathrm{w}$ time point of CIA mice, CIA mice were injected intraperitoneally (i.p.) with anti-IL1 $\beta$ or anti-TNFa + anti-IL $1 \beta$ or isotype IgG three times within a week, and the total amount of three consecutive injections was $0.9 \mathrm{mg}$ for each antibody. The mice were sacrificed at 10-week time point and TFH and TFR cells from the spleen and peripheral blood (PB) were obtained for experiments.

\subsection{Flow cytometry analysis}

Spleens were acquired and ground into cell suspensions and lymphocytes were isolated by Separation medium (Dakewei Biotech Co., Ltd. Shenzhen, China). Lymphocytes were then stained for surface markers for 30 min in PBS, including anti-CD4 PE-Cy7, anti-CXCR5 Alexa Fluo 647, anti-PD-1 APC-Cy7, anti-ICOS FITC, anti-TIGIT PE and anti-CD226 PE-Cy7(Biolegend, San Diego, CA, USA). Cells were then 
washed twice with PBS, and intracellular staining was performed with the FoxP3/Transcription Factor Staining Buffer Kit (TONBO Biosciences, San Diego, CA, USA), according to the manufacturer's instructions. After fixation and permeabilization, anti-FoxP3 PerCP-eFluor710 (eBioscience, Thermo Fisher Scientific, San Diego, CA, USA) were added and incubated for $30 \mathrm{~min}$. To detect cytokine production by lymphocytes, cells were cultured with PMA $(50 \mathrm{ng} / \mathrm{ml})$ and ionomycin $(1 \mu \mathrm{g} / \mathrm{ml})$ (PeproTech, Rocky Hill, NJ, USA) for $5 \mathrm{~h}$ with brefeldin A (1000x, Biolegend, San Diego, CA, USA) in RPMI 1640 medium containing 10\% FCS. After staining for surface markers, including anti-CD4 APC-Cy7 and anti-CXCR5 Alexa Fluo 647(Biolegend), cells were also fixed and permeabilized, and then incubated with antibodies including anti-FoxP3 PerCP-eFluor710(ebioscience), anti-IFNy PECy7, anti-IL-17A FITC, anti-IL-21 PE, antiIL-10 PE, anti-IL-4 PECy7 and anti-TNFa FITC(Biolegend). Samples were analyzed on a FACSCanto using Diva software (BD Biosciences).

\subsection{In vitro cell culture}

Totally $5 \times 10^{4}$ spleen $\mathrm{CD} 4^{+} \mathrm{CXCR} 5^{+} \mathrm{CD} 19^{-} \mathrm{GITR}^{-}$TFH cells from 10 -week time point $\mathrm{CIA}$ mice and control mice were plated with $5 \times 10^{4} \mathrm{CD} 19^{+} B$ cells (from spleen of CIA mice) and anti-CD3 $(2 \mu \mathrm{g} / \mathrm{ml}$ ) plus anti$\mathrm{lgM}(5 \mu \mathrm{g} / \mathrm{ml})$ for 5 days in RPMl 1640 medium containing $10 \% \mathrm{FBS}$, cultured at $37^{\circ} \mathrm{C}$ and $5 \%$ carbon dioxide. For TFR cell suppression assays, $1.5 \times 10^{4}$ spleen $\mathrm{CD} 4^{+} \mathrm{CXCR} 5^{+} \mathrm{CD} 19^{-} \mathrm{GITR}^{+}$TFR cells from $10-$ week time point CIA mice and control mice were added to the wells along with TFH and B cells (both from spleen of CIA mice). All cells were sorted by a FACSAria II flow cytometer (BD Biosciences), and the purity of each cell subset was verified to be greater than $97 \%$. Cells and supernatant were harvested and analyzed 5 days later. CD19+B cells were surface stained for GL7 and intracellularly stained for IgG1. IgG concentrations in supernatant were measured by ELISA.

\subsection{Statistical Analysis}

Results between different groups were compared using student's t test. All analyses were carried out using the Prism software (GraphPad Software, San Diego, CA). P values less than 0.05 are regarded as significant.

\section{Results}

\subsection{The TFH and TFR cells in the germinal center of the spleen and peripheral blood showed a gradual increase in the CIA mouse model.}

The CIA model was established by immunizing DBA/1 mice with type II collagen and CFA. The model was successfully established according to the clinical scores (Fig. 1A\&B). The levels of TFH and TFR in the spleen and peripheral blood of CIA mice and control mice were analyzed at different time points after the first immunization (week 0). As shown in Fig. 1, at the early stage of modeling (5 weeks), the TFH levels in spleen of CIA mice and the controls were not significantly different, but as the weeks increased, the TFH levels were significantly higher than those of the controls, for both percentages and absolute numbers (Fig. 1C\&D). In terms of TFR levels, the result showed that the difference in the early stage of 
modeling is not large, but as the age increased, TFR levels in spleen of CIA mice were significantly higher than controls (Fig. 1C\&D). We studied TFH and TFR levels in peripheral blood and the changes were in accordance with situations in spleens (Fig. 1E). We also compared TFH to TFR ratios and found that there was no significant difference between CIA mice and the controls, suggesting that TFH and TFR Raise in parallel. This part of the results suggests that TFH and TFR levels gradually increase in the germinal center during the process of RA, and the changes in peripheral blood and germinal center TFH and TFR are consistent.

\subsection{The spleen and peripheral blood TFH and TFR cells of CIA mice treated with anti-TNFa and anti-IL-1 $\beta$ neutralizing antibodies showed a significant decrease.}

We next used neutralizing antibodies of inflammatory cytokines for CIA treatment. The treatment options selected include anti-TNFa + anti-IL $1 \beta$ and anti-IL-1 $\beta$ alone. Because antibodies to different inflammatory factors play different roles, we used two different strategies to treat $\mathrm{CIA}$ mice separately, and also aimed to compare the differences in their therapeutic effects. We chose $10 \mathrm{w}$ as the termination time point to compare with the CIA mice treated with isotype IgG, because 10-week time point showed highest clinical scores and the level changes of TFH and TFR were also the most significant. We found that the clinical scores were significantly decreased after antibody injection, but there was no significant difference between mice injected with anti-TNFa + anti-IL1 $\beta$ and anti-IL-1 $\beta$ alone (Fig. 2A\&B). After using the two regimens, the spleen TFH and TFR of CIA mice showed a significant downward trend, for both (Fig. $2 \mathrm{C}$ ), while the peripheral blood TFH and TFR also significantly decreased (Fig. 2D). But there is no significant difference between the two different treatment options. We also compared TFH to TFR ratios and found that there was no significant difference among three groups (Fig. 2E). The results showed that TFH and TFR in CIA mice treated with inflammatory factor neutralizing antibodies were significantly reduced, suggesting that the consequence of this anti-inflammatory immunotherapy method is that the TFH/TFR system in mice is consistently reduced.

\subsection{TIGIT + and TIGIT + CD226-TFH cells in the spleen of CIA mice were significantly elevated and their levels recovered after inflammatory factor antibody treatment.}

To further clarify TFH and TFR subpopulation changes in the germinal center and peripheral blood of CIA mice, the main functionally related marker molecules were analyzed in detail. As shown in Fig. 3A\&B, in the TFH cells of the spleen in CIA mice, the expression of T cell immunoglobulin and ITIM domain (TIGIT) was always significantly higher than that of the control group, and the ratio of TIGIT + CD226- subset was also significantly higher than that of the control group. TFH in peripheral blood showed the same trend, but for TFR cells, there was no significant difference (data not shown). It was reported that TIGIT + TFH cell were with a stronger ability to help B cells[28], so the increase in TFH levels reflects its enhanced function. In terms of treatment, it' s interesting that after neutralizing antibody treatment, the ratios of TIGIT + and TIGIT + CD226 + in TFH cells were significantly reduced (Fig. 3C).

\subsection{The expression of PD-1 and ICOS on the surface of TFH and TFR cells in the spleen of CIA mice was significantly increased.}


Considering that CXCR5 alone may not fully represent mouse peripheral TFH and TFR cells, we further studied the changes in the expression levels of PD-1 and ICOS on TFH and TFR cells based on the previous results. We measured PD- 1 and ICOS on the surface of TFH and TFR cells in CIA mice. As shown in Fig. 4, PD-1+, ICOS + and PD-1 + ICOS + percentages in CD 4 + CXCR5 + FoxP3-TFH cells in the spleen of $\mathrm{CIA}$ mice increased significantly compared with the controls. These molecules are considered to indicate functions of TFH cells, so these results suggest that the function of TFH cells in the germinal center of the spleen of CIA mice were increased. In addition, we also found that TFR cells in the spleen of CIA mice also showed a significant increase (Fig. 4), and the changes were consistent with TFH cells.

\subsection{Treatment with neutralizing antibodies to inflammatory factors can restore the elevated PD-1 and ICOS expression in spleen TFH and TFR cells of CIA mice.}

We further explored the effect of inflammatory factor antibody treatment on PD-1 and ICOS on the surface of TFH and TFR in CIA mice. The results showed that the percentages of PD- $1+$ and PD- $1+$ ICOS + cells in TFH cells decreased significantly after treatment, while the percentages of ICOS + cell decreased significantly after treatment with IL-1 $\beta$ antibody alone (Fig. 5). For TFR cells, all the three subsets were decreased significantly after being treated by the two treatment options. And PD-1 + percentages were also lower after treatment with IL-1 $\beta$ antibody alone than treated with anti-TNFa plus anti-IL-1 $\beta$ (Fig. 5). These results suggested that the expression of TFH and TFR marker PD-1 and ICOS were significantly changed after inflammatory factor antibody treatment.

\subsection{The ability of TFH to secrete IL-21 and TFR to secrete IL-10 in the spleen of CIA mice is relatively enhanced, and the inflammatory factor neutralizing antibody treatment can restore its secretion ability.}

To further study the ability of TFH and TFR to secrete cytokines in the germinal center of CIA mice, we cultured and stimulated lymphocytes and detect the ability of TFH and TFR cells to secrete IL-4, IFNy, IL17, IL-21, IL-10 and TNFa. The results are shown in Fig. 6. For TFH cells, the secretion of IL-21 was significantly higher than that of the control group, which suggests that the function of TFH is enhanced in CIA mice. In terms of TFR, the secretion ability of IL-10 in CIA mice is significantly higher than that of the control group. For secretion of other cytokines, there was no difference. In terms of treatment, the levels of IL-21 and IL-10 secreted by TFH and TFR cells after treatment showed a significant decreasing trend, indicating that inflammatory cytokine antibody treatment resulted in a significant decrease in the ability to secrete cytokines for TFH and TFR cells, and its immune function is seriously affected.

\subsection{The functions of TFH and TFR cells in the spleen of CIA mice are both enhanced and could be recovered by neutralizing antibodies to inflammatory factors.}

In order to directly prove the functional changes of TFH and TFR cells in the spleen germinal center of CIA mice, we used an in vitro co-culture system. TFH cells of different sources were co-cultured with $B$ cells (from CIA mice) to test the function of TFH, and TFR cells of different sources were co-cultured with TFH $+B$ cells (from CIA mice) to test the function of TFR. We measured the functional changes of TFH and TFR in regulating antibody secretion by detecting the percentages of $G L 7+\lg G 1+B$ cells in the culture 
system and the levels of IgG in the supernatant. The results showed that the function of TFH cells derived from CIA mice to help B cells and the function of TFR cells to inhibit TFH cells were both relatively enhanced (Fig. 7A-C).

We also tested the functions of TFH and TFR cells in CIA mice treated with inflammatory cytokine antibodies, and the results showed that the functions of TFH and TFR cells were relatively reduced after antibody treatment (Fig. 7D\&E). The difference between the two different treatment options (anti-TNFa + anti-IL1 $\beta$ and anti-IL-1 $\beta$ alone) is not significant enough.

\section{Discussion}

In this study, we used CIA model to study the expression and function changes of TFH and TFR subsets in spleen germinal centers and clarify their significance in the pathogenesis of rheumatoid arthritis. We found that TFH and TFR cells were increased and their functions were enhanced, and the functional subset changes were in consistent with in vitro function assay results. After the treatment by inflammatory factor antibodies, TFH and TFR subsets and their functions were found to be recovered. This is the first systematic study of the functions and significance of TFH and TFR in the germinal centers of rheumatoid arthritis through animal models, which has important significance and clinical reference value.

TFH cells and TFR cells play important roles in numerous autoimmune diseases, such as SLE and RA[20, 21]. Although there have been reports on the significance of peripheral TFH and TFR in patients with rheumatoid arthritis, the changes and significance of TFH and TFR in the germinal center of rheumatoid arthritis are still unclear. We found that TFH and TFR cells in the germinal center of the spleen and peripheral blood showed a gradual increase in the CIA mouse model. This also suggests that the changes of TFH and TFR in the germinal center have a potential impact on the changes of peripheral blood TFH and TFR cells. According to our results, the increase in spleen TFH and TFR cells in CIA mice are consistent with the changes in peripheral blood of RA patients. But as for treatment, TFH and TFR were both decreased, which is inconsistent with the results of elevated TFR cells in RA patients with stable remission [21]. This may be related to differences in treatment options. The main treatment plan for the RA patients we studied is methotrexate, and its pharmacological mechanism is completely different from the use of inflammatory cytokine neutralizing antibodies. This indicates that different treatment strategies have different effects on patients' TFH and TFR levels. This difference provides an important reference for different treatment options in the future.

In this study, we conducted an in-depth analysis of the phenotypic changes of TFH and TFR cells in the spleen of CIA mice. Circulating T follicular helper cells expressing TIGIT were reported to exhibit strong Bcell help functions [28]. Considering that CD226 could and compete with TIGIT [29], The downregulation of CD226 relatively enhances the function of TIGIT on T cell subsets, so it is speculated that TIGIT + CD226-TFH cells have a relatively stronger function of helper B cells. We found that TIGIT + and TIGIT + CD226-TFH cells in the spleen of CIA mice were significantly elevated, which suggest that the function of 
TFH cells may be relatively enhanced. ICOS and PD- 1 are key molecules expressed on TFH cells, of which ICOS is a molecule expressed in T cells after activation [30, 31]. ICOS is essential for TFH development and plays important roles in B cells maturation and activation by combining ICOS-L expressed on B cells [32]. Circulating ICOS + PD-1 + TFH cells had been reported to play important roles in several diseases [33, 34]. PD-1+, ICOS + and PD-1 + ICOS + percentages in CD4 + CXCR5 + FoxP3-TFH cells in the spleen of CIA mice were found to be increased significantly, suggesting that TFH function was enhanced, which was also been proved by IL-21 secretion measurement and in vitro function assays.

The ability of TFH to secrete IL-21 and TFR to secrete IL-10 in the spleen of CIA mice was measured to be relatively enhanced. Interleukin (IL)-21 has been reported to play a role in TFH cell formation and in B cell growth and survival [35-37]. The ability of TFH to secrete IL-21 in the spleen of CIA mice was relatively enhanced, which suggests that TFH cells have enhanced functions in the process of CIA. IL-21 upregulating expression may promote the function of TFH in CIA. IL-10-producing TFR cells were reported to have a major role in maintaining the GC reaction and thus act as "helper cells" [38, 39]. In our research, we found that the ability of TFR cells to secrete IL-10 was relatively enhanced, but in terms of functional studies, it was found that the ability of TFR cells to inhibit TFH was also enhanced, which was manifested as a decrease in the level of antibodies secreted by $B$ cells. This contradiction can be explained that TFR cells not only function through IL-10, but also directly function on TFH cells. Although the level of IL-10 + TFR cells increases, the final result is an increase in TFR inhibition function.

The PD-1/PD-L1 pathway was reported to play a critical role in TFR generation and function, and PD1/PD-L1 signal could inhibit TFR differentiation and controls their suppressive ability [16]. In our research, we found PD-1 + TFR cells were increased in spleen of CIA mice, but in in-vitro function assay, TFR cell function was relatively enhanced. This inconsistency may be due to the fact that although the expression level of PD1 on TFR can reflect the activation level of the signal pathway to a certain extent, the two may not be completely consistent. In addition, we also found that the expression of ICOS is up regulated in the spleen TFR cells of CIA mice. However, the detailed function of ICOS in TFR expression has not been reported and needs to be further elaborated in future studies.

Our research focuses on the impact of inflammatory factor neutralizing antibody therapy on TFH and TFR. According to previous reports, we selected two different treatment options, including anti-TNFa plus anti-IL-1 $\beta$ and anti-IL-1 $\beta$ alone $[40,41]$. The results showed that after treatment with anti-TNFa and anti-IL$1 \beta$ or IL-1 $\beta$ alone, the levels, subpopulations, and functions of TFH and TFR were significantly restored. This result indicates that inflammatory factors play an important role in the increase and function enhancement of TFH and TFR in CIA mice. According to the results, the difference between the two treatment options is not obvious, which suggests that the IL-1 $\beta$ antibody plays a relatively greater role in the treatment. As to why the use of anti-IL1 $\beta$ and anti-TNFa can significantly restore the dys-regulated TFH and TFR cells in CIA mice, it will require very complicated work to clarify. The first possible reason is that they significantly changed the in vivo environment of inflammatory factors, and the changes of these inflammatory factors further caused changes in the cytokine network, which ultimately affected the differentiation and function of TFH and TFR cells. In addition, the use of inflammatory cytokine 
antibodies can significantly act on cells with corresponding receptors, which may include $T$ cells themselves, as well as B cells and antigen-presenting cells that interact with $T$ cells. The interaction of these cells with antibodies leads to changes in cell behavior related to TFH and TFR, and further affects cell level and function.

There are still many shortcomings in our study. One important point is that our research focused on the changes in T cell subpopulations, but we did not pay more attention to the dynamic changes of B cells and antibody secretion in CIA mice. However, we quantitatively measured the B cells through in vitro culture function assays as compensation. The dynamic changes of B cells in vivo will be as a further research direction in future. The selection of healthy mice of the same age as the control of the CIA model may be one of the controversies in this study, but the mice with other treatment methods are also less representative of the situation of healthy individuals, e.g., only CFA is injected. So healthy mice of the same age can only be used as the control group. There are methodological limitations, but in order to study the TFH and TFR cells in the germinal center of the spleen, this can only be done. Although the CIA model is a classic RA animal model, it still needs further verification in the patient population. That is still one question whether the changes in TFH and TFR are factors that promote the onset of RA or are passive changes in the onset of RA. However, the current experimental results cannot answer this issue, and it need to be clarified in the future.

\section{Conclusions}

To conclude, in this study we demonstrated that in the pathogenesis of rheumatoid arthritis, TFH and TFR cells in the germinal center are increased and their functions are enhanced. Additionally, after the treatment by inflammatory factor antibodies, TFH and TFR subsets and their functions can be significantly recovered. The discovery about TFH and TFR cell subset and function changes in RA pathogenesis provides novel insight into the mechanism of RA, which may have potential clinical values for RA treatment.

\section{Abbreviations}

ARRIVE, Animal Research: Reporting of in Vivo Experiments guidelines; AS, ankylosing spondylitis; CIA, collagen-induced arthritis; ELISA, enzyme-linked immunosorbent assay; FCS, fetal calf serum; FoxP3, forkhead box P3; ICOS, inducible co-stimulator; IFN, interferon; IL, interleukin; MTX, Methotrexate; PD-1, positive programmed cell death 1; PE, phycoerythrin; PMA, phorbol 12-myristate 12-acetate; RA, rheumatoid arthritis; SLE, systemic lupus erythematosus; TFH, follicular helper T cells; TFR, follicular regulatory T cells; TIGIT, T cell Ig and ITIM domain; TNF, tumor necrosis factor; Treg, regulatory T cells; UC, ulcerative colitis.

\section{Declarations}

\subsection{Ethical approval and consent to participate}


All mice received human care and the research protocol complied with the Animal Research: Reporting of in Vivo Experiments (ARRIVE) guidelines, with approval from ethical board of Peking University People's Hospital.

\subsection{Consent for publication}

Every author has known and agreed to send and publish this paper to arthritis research \& therapy

\subsection{Availability of supporting data}

All supporting data in this study are available from the corresponding author on reasonable request.

\subsection{Competing interests}

There are no competing interests in relation to the work described.

\subsection{Funding}

This work was supported by grants from National Natural Science Foundation of China (81871230), Peking University Medicine Fund of Fostering Young Scholars' Scientific \& Technological Innovation (BMU2021PY008), Peking University People's Hospital Scientific Research Development Funds (RDT 2020-01) and the Fundamental Research Funds for the Central Universities

\subsection{Authors' contributions}

$\mathrm{CL}$ designed research studies and wrote the manuscript, $X Z$ and $C L$ did the experiments, TL, RK and ML acquired data, MZ, LX and QX analyzed data and contributed to methods. YS and SL provided technical assistance and part of the design of the study.

\subsection{Acknowledgements}

We thank the Department of Animal Unit of Peking University People's Hospital for their service.

\section{Disclosures}

The authors declare that they have no competing interests.

\section{References}

1. Mclnnes IB, Schett G: The pathogenesis of rheumatoid arthritis. The New England journal of medicine 2011, 365(23):2205-2219.

2. Conigliaro P, Chimenti MS, Triggianese P, Sunzini F, Novelli L, Perricone C, Perricone R: Autoantibodies in inflammatory arthritis. Autoimmunity reviews 2016, 15(7):673-683. 
3. Li ZG: A new look at rheumatology in China-opportunities and challenges. Nature reviews Rheumatology 2015, 11(5):313-317.

4. Wang J, Shan Y, Jiang Z, Feng J, Li C, Ma L, Jiang Y: High frequencies of activated B cells and T follicular helper cells are correlated with disease activity in patients with new-onset rheumatoid arthritis. Clinical and experimental immunology 2013, 174(2):212-220.

5. Niu Q, Cai B, Huang ZC, Shi YY, Wang LL: Disturbed Th17/Treg balance in patients with rheumatoid arthritis. Rheumatology international 2012, 32(9):2731-2736.

6. Wang W, Shao S, Jiao Z, Guo M, Xu H, Wang S: The Th17/Treg imbalance and cytokine environment in peripheral blood of patients with rheumatoid arthritis. Rheumatology international 2012, 32(4):887-893.

7. Samson M, Audia S, Janikashvili N, Ciudad M, Trad M, Fraszczak J, Ornetti P, Maillefert JF, Miossec P, Bonnotte B: Brief report: inhibition of interleukin-6 function corrects Th17/Treg cell imbalance in patients with rheumatoid arthritis. Arthritis and rheumatism 2012, 64(8):2499-2503.

8. Vinuesa CG, Linterman MA, Yu D, MacLennan IC: Follicular Helper T Cells. Annual review of immunology 2016, 34:335-368.

9. Lüthje K, Kallies A, Shimohakamada Y, Belz GT, Light A, Tarlinton DM, Nutt SL: The development and fate of follicular helper T cells defined by an IL-21 reporter mouse. Nature immunology 2012, 13(5):491-498.

10. Shulman Z, Gitlin AD, Targ S, Jankovic M, Pasqual G, Nussenzweig MC, Victora GD: T follicular helper cell dynamics in germinal centers. Science (New York, NY) 2013, 341(6146):673-677.

11. Szabo K, Papp G, Barath S, Gyimesi E, Szanto A, Zeher M: Follicular helper T cells may play an important role in the severity of primary Sjögren's syndrome. Clinical immunology (Orlando, Fla) 2013, 147(2):95-104.

12. Morita R, Schmitt N, Bentebibel SE, Ranganathan R, Bourdery L, Zurawski G, Foucat E, Dullaers M, Oh S, Sabzghabaei $N$ et al: Human blood CXCR5(+)CD4(+) T cells are counterparts of $T$ follicular cells and contain specific subsets that differentially support antibody secretion. Immunity 2011, 34(1):108-121.

13. He J, Tsai LM, Leong YA, Hu X, Ma CS, Chevalier N, Sun X, Vandenberg K, Rockman S, Ding Y et al: Circulating precursor CCR7(lo)PD-1(hi) CXCR5区 CD4区 T cells indicate Tfh cell activity and promote antibody responses upon antigen reexposure. Immunity 2013, 39(4):770-781.

14. Ma J, Zhu C, Ma B, Tian J, Baidoo SE, Mao C, Wu W, Chen J, Tong J, Yang M et al: Increased frequency of circulating follicular helper $T$ cells in patients with rheumatoid arthritis. Clinical \& developmental immunology 2012, 2012:827480.

15. Rasmussen TK, Andersen T, Hvid M, Hetland ML, Hørslev-Petersen K, Stengaard-Pedersen K, Holm CK, Deleuran B: Increased interleukin 21 (IL-21) and IL-23 are associated with increased disease activity and with radiographic status in patients with early rheumatoid arthritis. The Journal of rheumatology 2010, 37(10):2014-2020. 
16. Sage PT, Francisco LM, Carman CV, Sharpe AH: The receptor PD-1 controls follicular regulatory T cells in the lymph nodes and blood. Nature immunology 2013, 14(2):152-161.

17. Chung Y, Tanaka S, Chu F, Nurieva RI, Martinez GJ, Rawal S, Wang YH, Lim H, Reynolds JM, Zhou $\mathrm{XH}$ et al: Follicular regulatory T cells expressing Foxp3 and Bcl-6 suppress germinal center reactions. Nature medicine 2011, 17(8):983-988.

18. Fu W, Liu X, Lin X, Feng H, Sun L, Li S, Chen H, Tang H, Lu L, Jin W et al: Deficiency in T follicular regulatory cells promotes autoimmunity. The Journal of experimental medicine 2018, 215(3):815825.

19. Shan Y, Qi C, Zhao J, Liu Y, Gao H, Zhao D, Ding F, Wang J, Jiang Y: Higher frequency of peripheral blood follicular regulatory $\mathrm{T}$ cells in patients with new onset ankylosing spondylitis. Clinical and experimental pharmacology \& physiology 2015, 42(2):154-161.

20. Liu C, Wang D, Song Y, Lu S, Zhao J, Wang H: Increased circulating CD4(+)CXCR5(+)FoxP3(+) follicular regulatory $T$ cells correlated with severity of systemic lupus erythematosus patients. International immunopharmacology 2018, 56:261-268.

21. Liu C, Wang D, Lu S, Xu Q, Zhao L, Zhao J, Song Y, Wang H: Increased Circulating Follicular Treg Cells Are Associated With Lower Levels of Autoantibodies in Patients With Rheumatoid Arthritis in Stable Remission. Arthritis \& rheumatology (Hoboken, NJ) 2018, 70(5):711-721.

22. Brand DD, Latham KA, Rosloniec EF: Collagen-induced arthritis. Nature protocols 2007, 2(5):12691275.

23. Haleagrahara N, Miranda-Hernandez S, Alim MA, Hayes L, Bird G, Ketheesan N: Therapeutic effect of quercetin in collagen-induced arthritis. Biomedicine \& pharmacotherapy = Biomedecine \& pharmacotherapie 2017, 90:38-46.

24. Croft M, Benedict CA, Ware CF: Clinical targeting of the TNF and TNFR superfamilies. Nature reviews Drug discovery 2013, 12(2):147-168.

25. Strand V, Kimberly R, Isaacs JD: Biologic therapies in rheumatology: lessons learned, future directions. Nature reviews Drug discovery 2007, 6(1):75-92.

26. Dinarello CA, Simon A, van der Meer JW: Treating inflammation by blocking interleukin-1 in a broad spectrum of diseases. Nature reviews Drug discovery 2012, 11(8):633-652.

27. Alten R, Gomez-Reino J, Durez P, Beaulieu A, Sebba A, Krammer G, Preiss R, Arulmani U, Widmer A, Gitton $X$ et al: Efficacy and safety of the human anti-IL-1 $\beta$ monoclonal antibody canakinumab in rheumatoid arthritis: results of a 12-week, Phase II, dose-finding study. BMC musculoskeletal disorders 2011, 12:153.

28. Godefroy E, Zhong H, Pham P, Friedman D, Yazdanbakhsh K: TIGIT-positive circulating follicular helper $\mathrm{T}$ cells display robust B-cell help functions: potential role in sickle cell alloimmunization. Haematologica 2015, 100(11):1415-1425.

29. Anderson AC, Joller N, Kuchroo VK: Lag-3, Tim-3, and TIGIT: Co-inhibitory Receptors with Specialized Functions in Immune Regulation. Immunity 2016, 44(5):989-1004. 
30. Hutloff A, Dittrich AM, Beier KC, Eljaschewitsch B, Kraft R, Anagnostopoulos I, Kroczek RA: ICOS is an inducible T-cell co-stimulator structurally and functionally related to CD28. Nature 1999, 397(6716):263-266.

31. Locci M, Havenar-Daughton C, Landais E, Wu J, Kroenke MA, Arlehamn CL, Su LF, Cubas R, Davis MM, Sette A et al: Human circulating PD-1+CXCR3-CXCR5+ memory Tfh cells are highly functional and correlate with broadly neutralizing HIV antibody responses. Immunity 2013, 39(4):758-769.

32. Sefer AP, Charbonnier LM, Kasap N, Akcam B, Demirkol YK, Eltan SB, Ozen A, Karakoc-Aydiner E, Baris S: A Patient with Novel ICOS Mutation Presented with Progressive Loss of B Cells. Journal of clinical immunology 2021, 41(1):251-255.

33. Long Y, Zhao X, Liu C, Xia C, Liu C: Activated inducible co-stimulator-positive programmed cell death 1-positive follicular helper $T$ cells indicate disease activity and severity in ulcerative colitis patients. Clinical and experimental immunology 2020, 202(1):106-118.

34. Danger R, Chesneau M, Delbos F, Le Bot S, Kerleau C, Chenouard A, Ville S, Degauque N, Conchon S, Cesbron A et al: CXCR5(+)PD1(+)ICOS(+) Circulating T Follicular Helpers Are Associated With de novo Donor-Specific Antibodies After Renal Transplantation. Frontiers in immunology 2019, 10:2071.

35. Linterman MA, Beaton L, Yu D, Ramiscal RR, Srivastava M, Hogan JJ, Verma NK, Smyth MJ, Rigby RJ, Vinuesa CG: IL-21 acts directly on B cells to regulate Bcl-6 expression and germinal center responses. The Journal of experimental medicine 2010, 207(2):353-363.

36. Spolski R, Leonard WJ: Interleukin-21: basic biology and implications for cancer and autoimmunity. Annual review of immunology 2008, 26:57-79.

37. Gharibi T, Majidi J, Kazemi T, Dehghanzadeh R, Motallebnezhad M, Babaloo Z: Biological effects of IL-21 on different immune cells and its role in autoimmune diseases. Immunobiology 2016, 221(2):357-367.

38. Laidlaw BJ, Lu Y, Amezquita RA, Weinstein JS, Vander Heiden JA, Gupta NT, Kleinstein SH, Kaech $\mathrm{SM}$, Craft J: Interleukin-10 from CD4(+) follicular regulatory T cells promotes the germinal center response. Science immunology 2017, 2(16).

39. Xie MM, Dent AL: Unexpected Help: Follicular Regulatory T Cells in the Germinal Center. Frontiers in immunology 2018, 9:1536.

40. Williams RO, Marinova-Mutafchieva L, Feldmann M, Maini RN: Evaluation of TNF-alpha and IL-1 blockade in collagen-induced arthritis and comparison with combined anti-TNF-alpha/anti-CD4 therapy. Journal of immunology (Baltimore, Md : 1950) 2000, 165(12):7240-7245.

41. van den Berg WB, Joosten LA, Kollias G, van De Loo FA: Role of tumour necrosis factor alpha in experimental arthritis: separate activity of interleukin 1 beta in chronicity and cartilage destruction. Annals of the rheumatic diseases 1999, 58 Suppl 1(Suppl 1):I40-48.

\section{Figures}


A

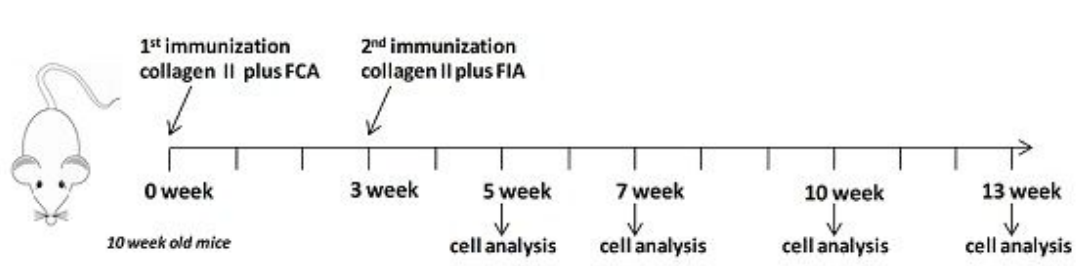

B

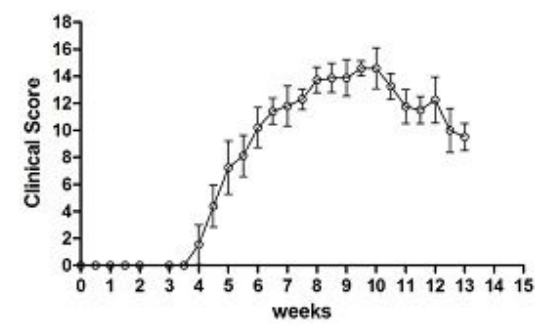

C
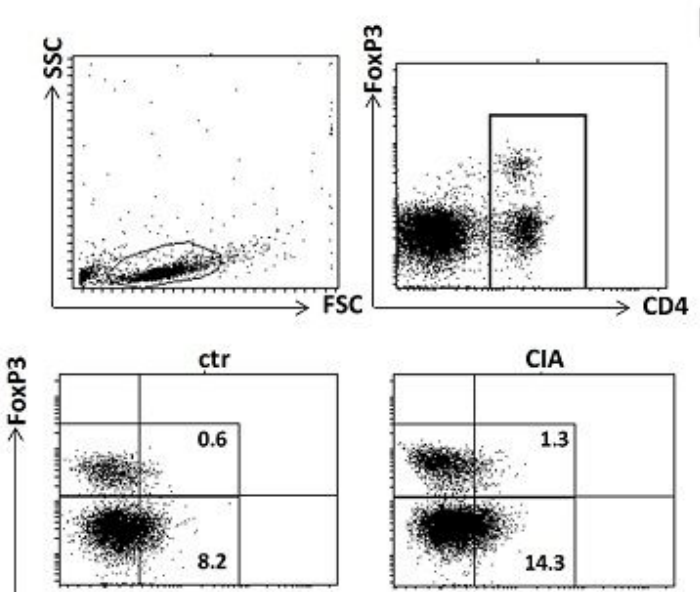

8.2

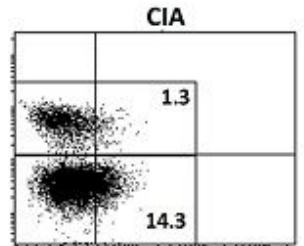

CXCR5
D
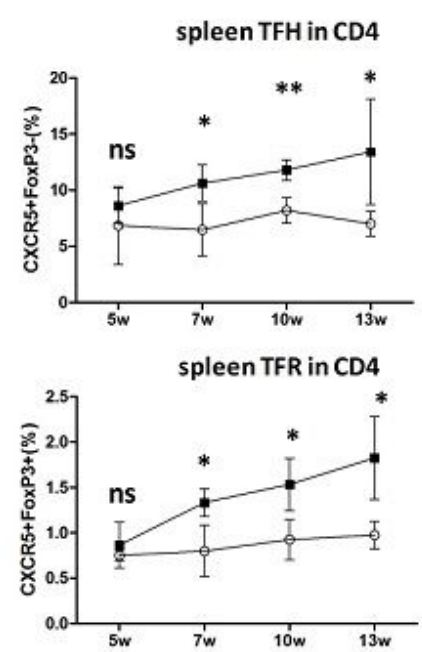

\# of spleen TFH

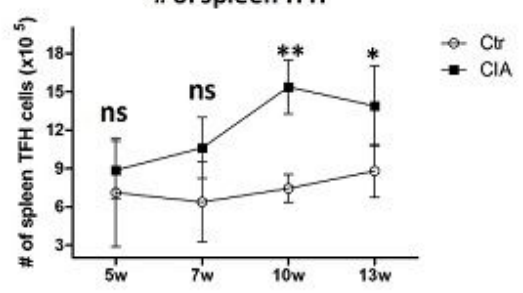

\# of spleen TFR

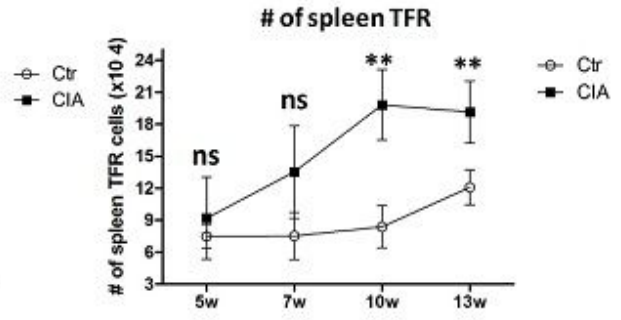

E circulating TFH in CD4 circulating TFR in CD4
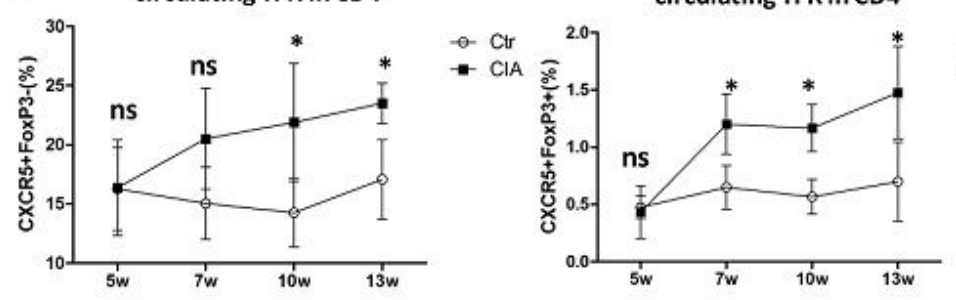

$\mathrm{F}$

$-\mathrm{Cr}$
$* \mathrm{CIA}$
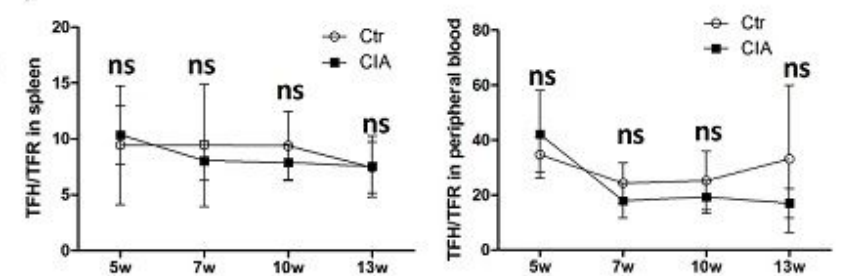

\section{Figure 1}

Analysis of level changes in peripheral TFH and TFR cells in collagen-induced arthritis mice. A. The collagen-induced arthritis (CIA) mouse model was applied to 10-week-old DBA/1 mice. After two immunizations ( $0 w, 3 w)$, the mice were sacrificed at different time points $(5 w, 7 w, 10 w, 13 w)$ and the lymphocytes in spleen and peripheral blood (PB) were separated. Healthy DBA/ 1 mice of the same age were used as control groups (Ctr). CD4+CXCR5+FoxP3+ TFR cells and CD4+CXCR5+FoxP3-TFH cells in the spleen and peripheral blood were detected by flow cytometry. B. Clinical score of the CIA mice were measured twice each week. C. Representative flow cytometry charts show percentages of TFH and TFR cells in the spleen CD4+ T cells of two groups of mice at the $10 \mathrm{w}$ point analyzed by flow cytometry. $\mathrm{D}$. The changes in the percentages(left) of TFH and TFR cells in the CD4+ lymphocytes and their absolute numbers(right)in the spleens of the two groups of mice were analyzed at different time points. E. Analysis of the percentages of TFH and TFR cells in the peripheral blood CD4+ lymphocytes of the two groups of mice. F. Ratios of TFH to TFR in spleen (left) and peripheral blood (right) of CIA and Ctr mice at different time points. Totally 3-4 mice in each group were tested at each time point and data are present as mean $\pm S D$. The experiment was repeated once and consistent results were obtained. ${ }^{*} p<0.01 ;{ }^{*} p<0.05$; ns, not significant. 
A

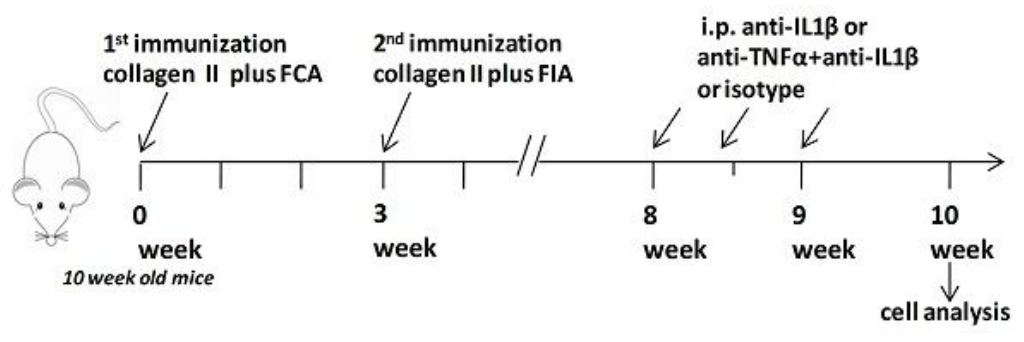

B

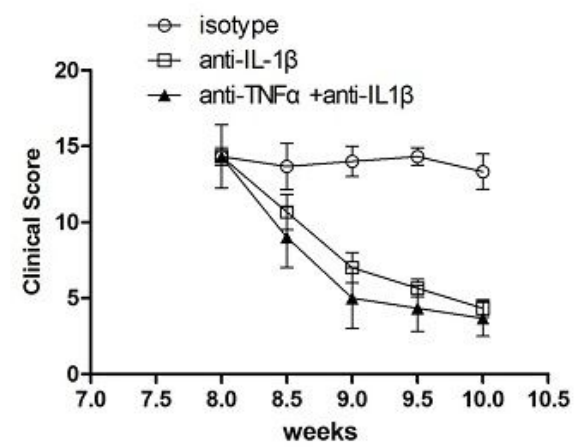

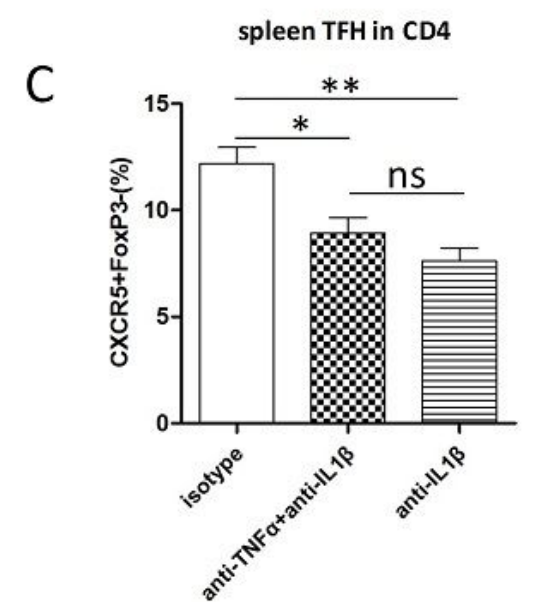
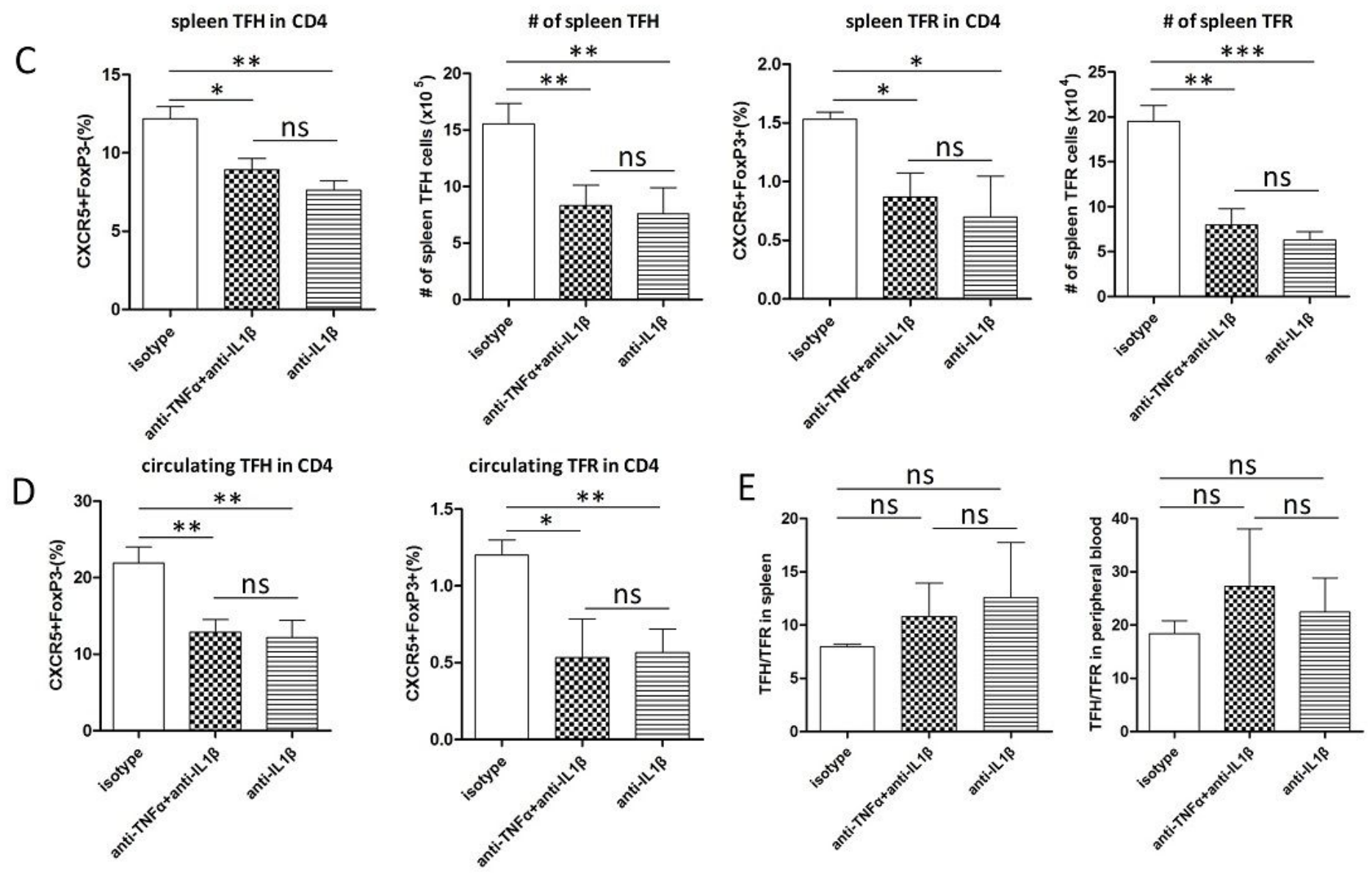

Figure 2

The effect of anti-inflammatory treatment on the levels of TFH and TFR of CIA mice. A. CIA mice were injected with anti-IL1 $\beta$ or anti-TNFa+anti-IL1 $\beta$ one week before the 10-week time point for three consecutive injections with a total amount of $0.9 \mathrm{mg}$, and the control mice were injected with isotype IgG. B. Clinical score of the CIA mice injected with anti-IL1 $\beta$ or anti-TNFa+anti-IL1 $\beta$ were measured twice each week after injection compared with the control mice injected with isotype IgG. C. The treated mice were sacrificed at the 10w time point and the spleen TFH and TFR cell percentages in CD4 lymphocytes and absolute numbers were analyzed and compared with control CIA mice. D. TFH and TFR cell percentages in circulating CD4 lymphocytes were analyzed and compared with control CIA mice. E. Ratios of TFH to TFR in spleen (left) and peripheral blood (right) of three groups of CIA mice were compared. There were 3 
mice in each group, and the experiment was repeated twice and reached consistent conclusions. Data are present as mean with SD. ${ }^{* *} \mathrm{p}<0.01,{ }^{*} \mathrm{p}<0.05$, ns, not significant.
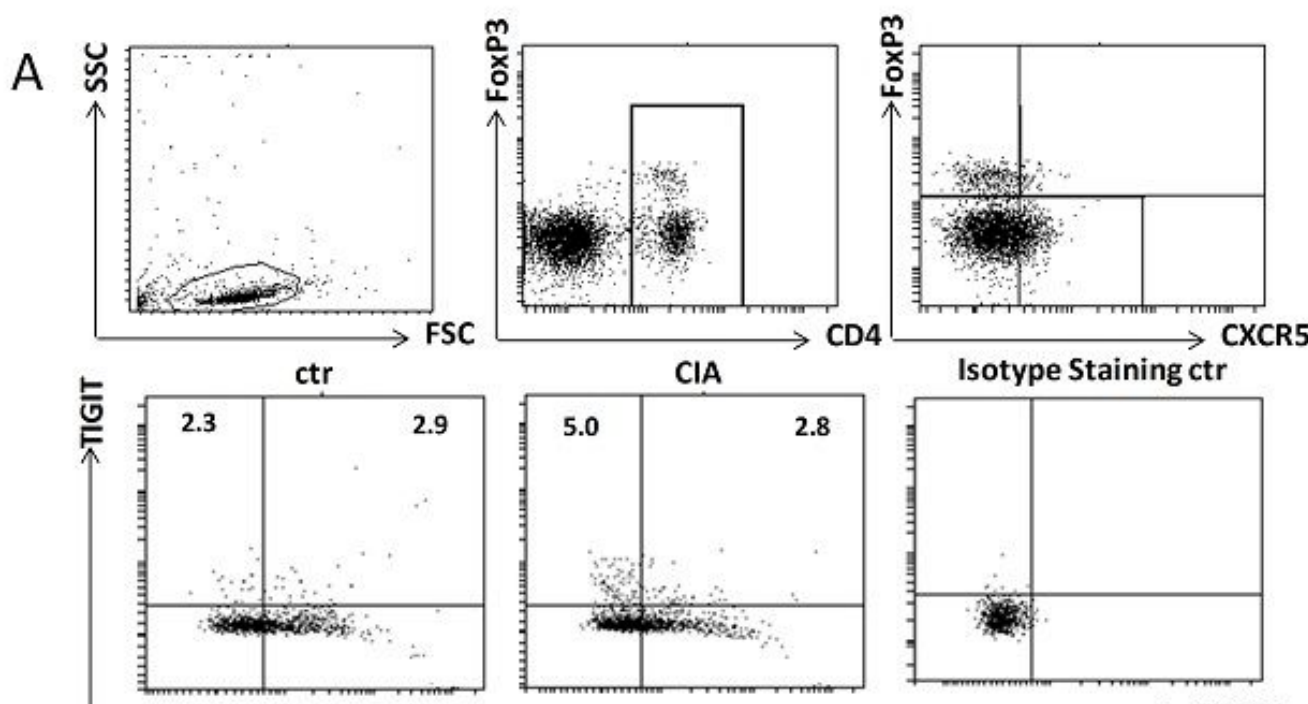

Isotype Staining ctr

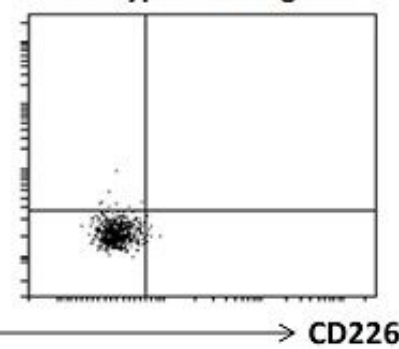

CD226
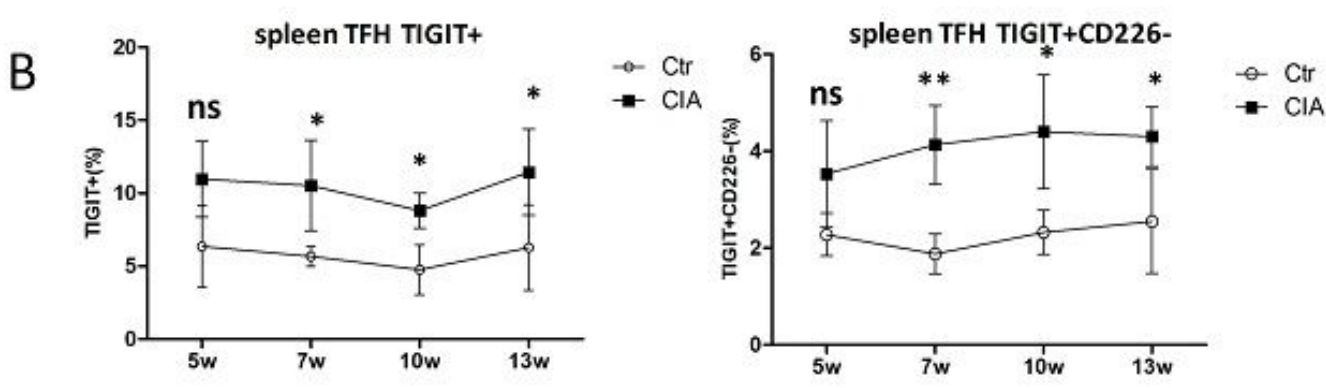

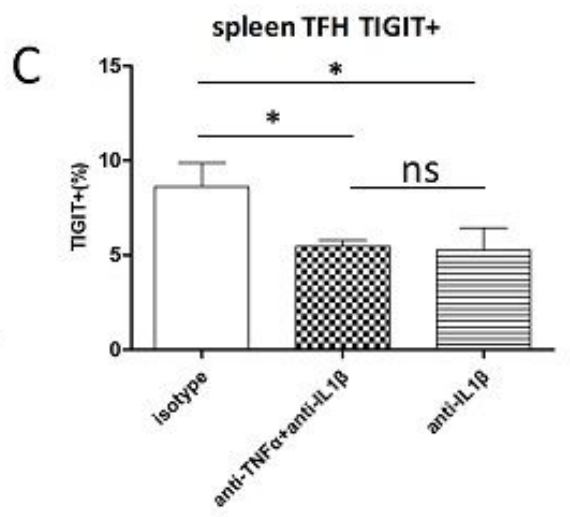

spleen TFH TIGIT+CD226-

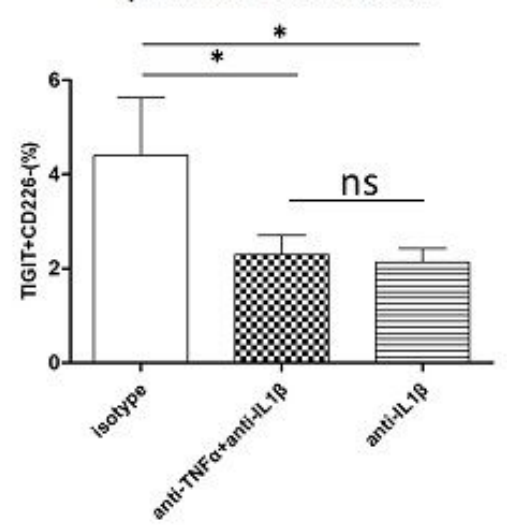

\section{Figure 3}

The expression level changes of TIGIT and CD226 on CIA mouse TFH cells. The spleen lymphocytes of CIA mice at different time points $(5 \mathrm{w}, 7 \mathrm{w}, 10 \mathrm{w}, 13 \mathrm{w})$ were obtained, and flow cytometry was used to detect the expression levels of CD226 and TIGIT on the surface of CD4+CXCR5+FoxP3-TFH cells. A. Representative flow cytometry plots show the division of TFH in the spleen CD4 cells of CIA mice at 10week point, and the expression of TIGIT and CD226 in TFH. The numbers indicate the percentages of corresponding quadrants in TFH cells. The isotype staining control was also shown. B. Comparison of the ratios of TIGIT+ and TIGIT+CD226- subsets in spleen TFH cells of CIA mice and healthy control DBA/1 mice at different time points after first immunization. Totally 3-4 mice in each group were tested at each time point and data are present as mean $\pm S D$. The experiment was repeated once and consistent results were obtained. ${ }^{\star \star} p<0.01 ;{ }^{*} p<0.05$; ns, not significant. C. Comparison of TIGIT+ and TIGIT+CD226cell percentages in TFH cells of CIA mice at 10 weeks injected with isotype IgG and CIA mice of the same age who have undergone two different anti-inflammatory treatments (anti-IL1 $\beta$ or anti-TNFa+anti-IL1 $\beta$ ). There were 3 mice in each group, and the experiment was repeated twice and reached consistent conclusions. Data are present as mean with SD. ${ }^{\star} p<0.05$; ns, not significant. 

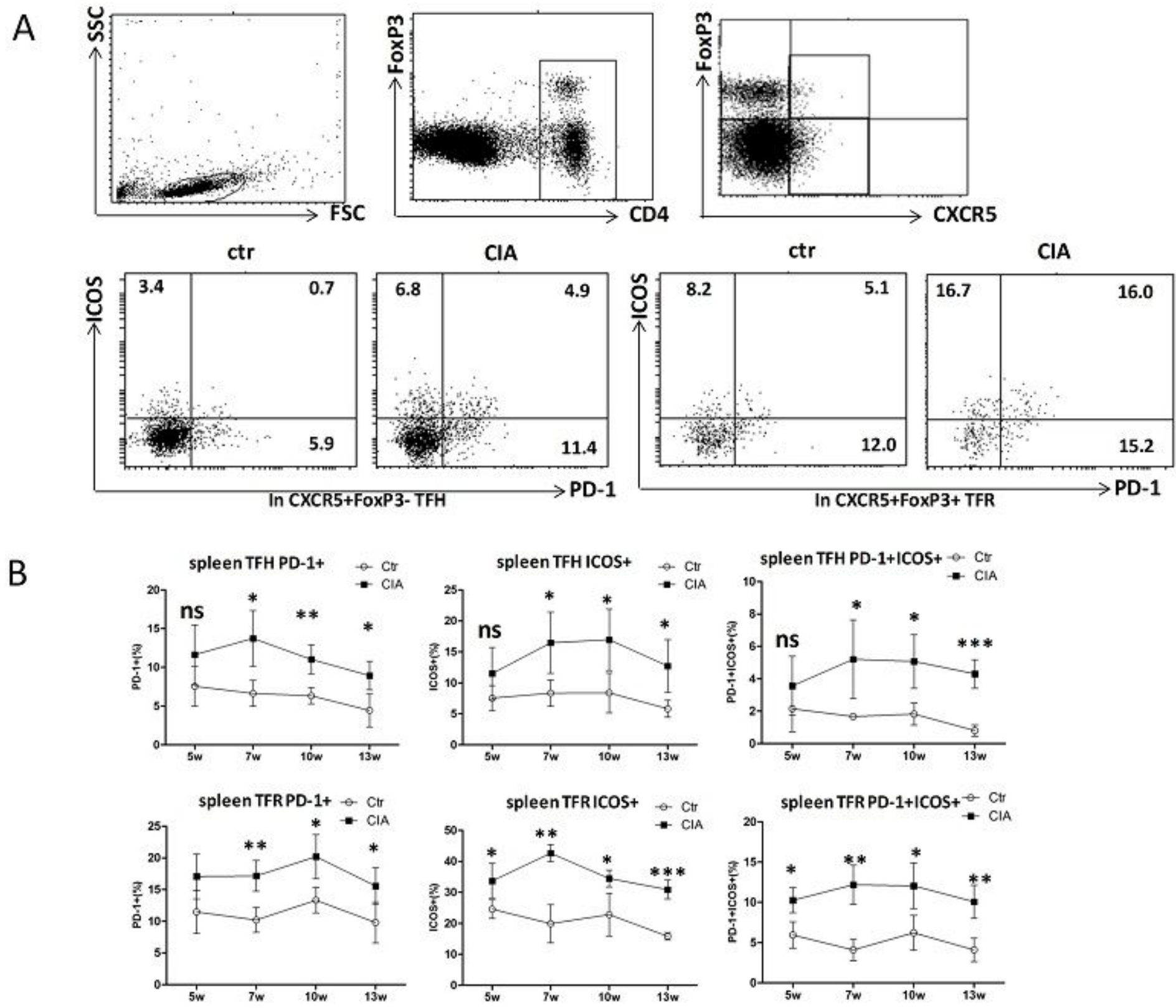

\section{Figure 4}

PD-1 and ICOS expression of TFH and TFR cells in the spleen of CIA mice. The spleen lymphocytes of CIA mice at different time points $(5 \mathrm{w}, 7 \mathrm{w}, 10 \mathrm{w}, 13 \mathrm{w})$ after the initial immunization were obtained, and the expression levels of PD- 1 and ICOS on the surface of CD4+CXCR5+ FoxP3-TFH cells and CD4+CXCR5+FoxP3+ TFR cells were detected by flow cytometry. A. Representative flow cytometry plots showing the expression analysis of PD-1 and ICOS on TFH and TFR cells of CIA mice and control mice of the same age at the 10-week time point. The numbers indicate the percentages of the corresponding quadrants in TFH or TFR cells. B. Comparison of the percentages of PD-1+, ICOS+ and PD-1+ICOS+ subsets in spleen TFH cells of CIA mice at different time points after the first immunization and healthy control DBA/1 mice with the same age. A total of 3-4 mice in each group were tested at each time point, and the data were expressed as mean $\pm S D$. The experiment was repeated once and got consistent results. $\star \star \star *<0.001 ; * \star p<0.01 ; * p<0.05 ;$ ns, not significant. 

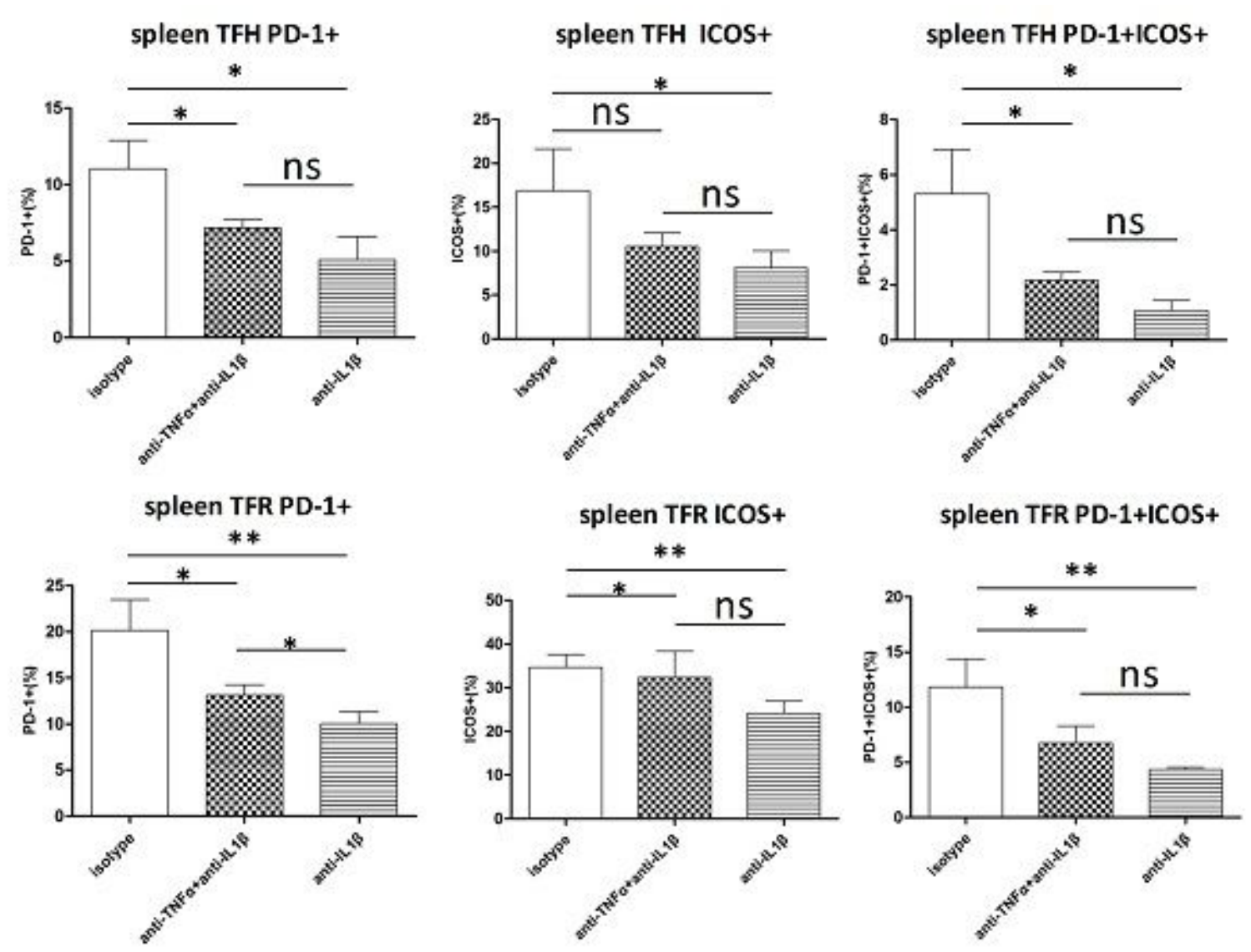

Figure 5

The expression changes of PD- 1 and ICOS on TFH and TFR cells with anti-inflammatory treatment. CIA mice were injected with anti-IL1 $\beta$ or anti-TNFa+anti-IL1 $\beta$ one week before the 10-week time point. The treated mice were sacrificed at the $10 \mathrm{w}$ time point and the PD-1+, ICOS+ and PD-1+ICOS+ subset percentages in spleen TFH and TFR cells were analyzed and compared with CIA mice of the same age injected with isotype $\operatorname{lgG}$. There were 3 mice in each group and the experiment was repeated twice and got consistent conclusions. The data are expressed as mean with SD. ${ }^{*} p<0.01$; ${ }^{*} p<0.05$; ns, not significant.

A
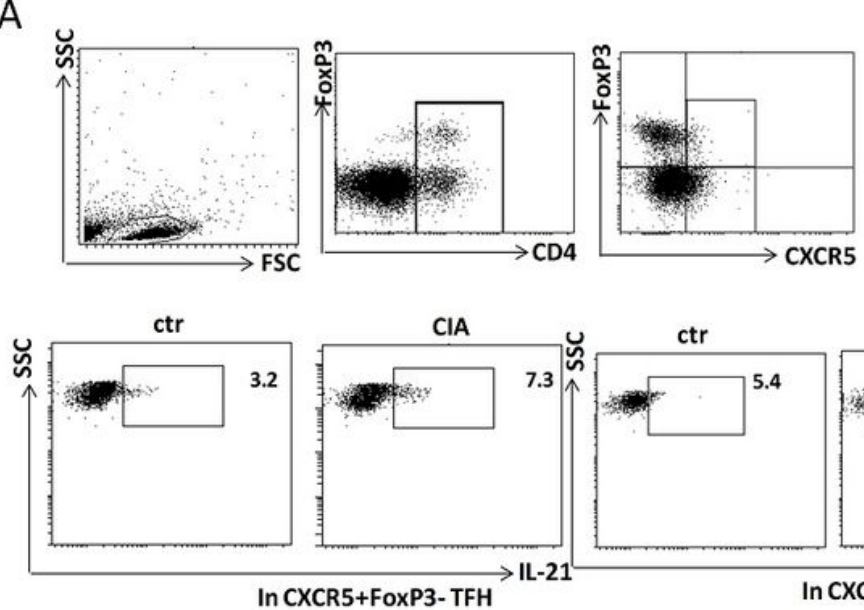

ctr

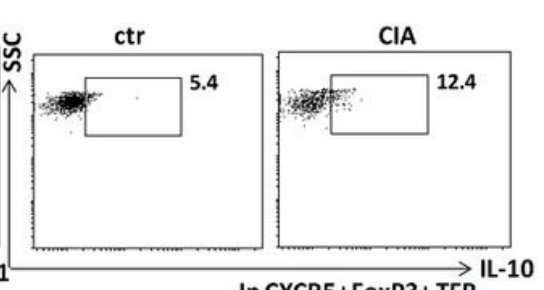

In CXCR5+FoxP3+TFR IL-10

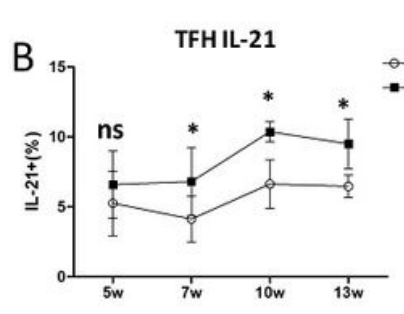

C

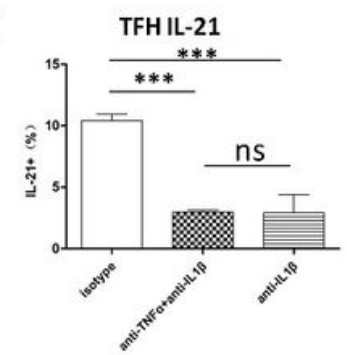

TFR IL-10
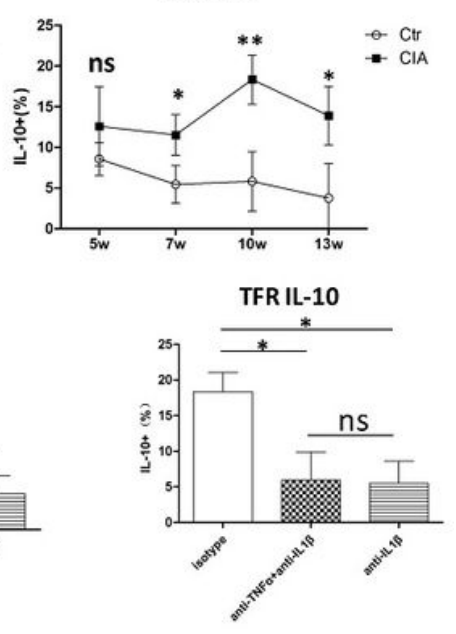


\section{Figure 6}

The secretion changes of IL-21 and IL-10 of TFH and TFR cells with anti-inflammatory treatment. Spleen lymphocytes from CIA mice and the control DBA/1 mice were cultured and stimulated with PMA $(50 \mathrm{ng} / \mathrm{ml})$ and ionomycin $(1 \mu \mathrm{g} / \mathrm{ml})$ for 5 hours with brefeldin $A$ in the medium. The cells were then harvested, stained with surface marker antibodies and then intracellularly stained with IL-21 and IL-10 and analyzed by flow cytometry. A. Representative flow cytometry dot plots showing the level of IL-21 in CD4+CXCR5+FoxP3-TFH cells and the expression of IL-10 in CD4+CXCR5+FoxP3+TFR cells. B.

Comparison of the ratios of IL-21+ TFH cells and IL-10+ TFR cells in spleens of CIA mice at different time points after the first immunization and healthy control DBA/1 mice with the same age. Each group contained 3-4 mice at each time point. The experiment was repeated once and consistent results were obtained. ** $\mathrm{p}<0.01$; ${ }^{\mathrm{p}}<0.05$; ns, not significant. C. Comparison of levels of IL-21+ TFH cells and IL-10+ TFR cells in CIA mice receiving two different anti-inflammatory treatments (anti-IL-1 $\beta$ or anti-TNFa+antiIL-1 $\beta$ ) and control CIA mice of the same age injected with isotype IgG. There were 3 mice in each group. The experiment was repeated once and got consistent results. The data are expressed as mean with SD. $\star \star \star ~ p<0.001 ; * p<0.05 ;$ ns, not significant. 
A

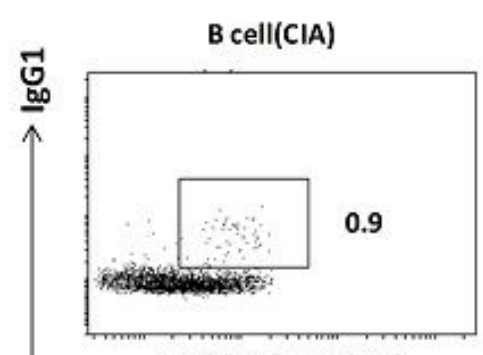

B cell(ClA $)+$ TFH(Ctr)

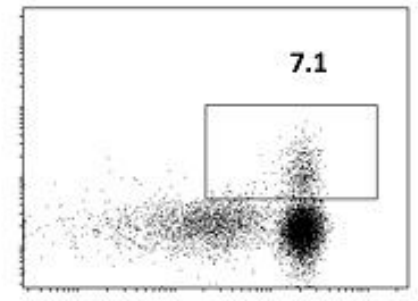

B cell $(\mathrm{CIA})+\mathrm{TFH}(\mathrm{CIA})+\mathrm{TFR}(\mathrm{Ctr})$

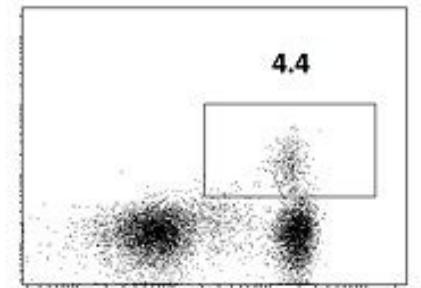

$B$ cell $(\mathrm{ClA})+\mathrm{TFH}(\mathrm{CIA})$

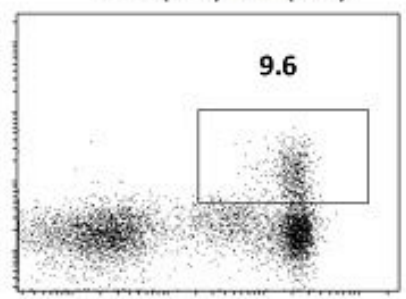

$B$ cell $(C \mid A)+T F H(C I A)+T F R(C I A)$

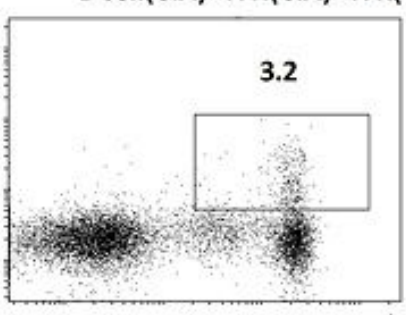

B

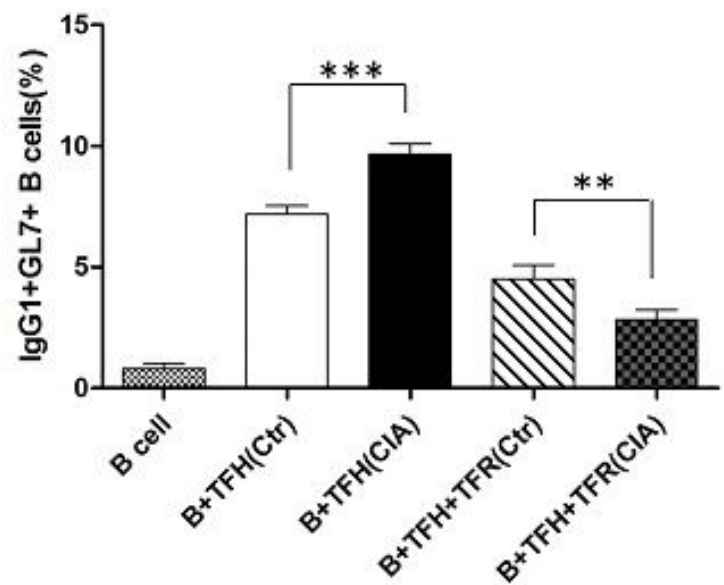

C

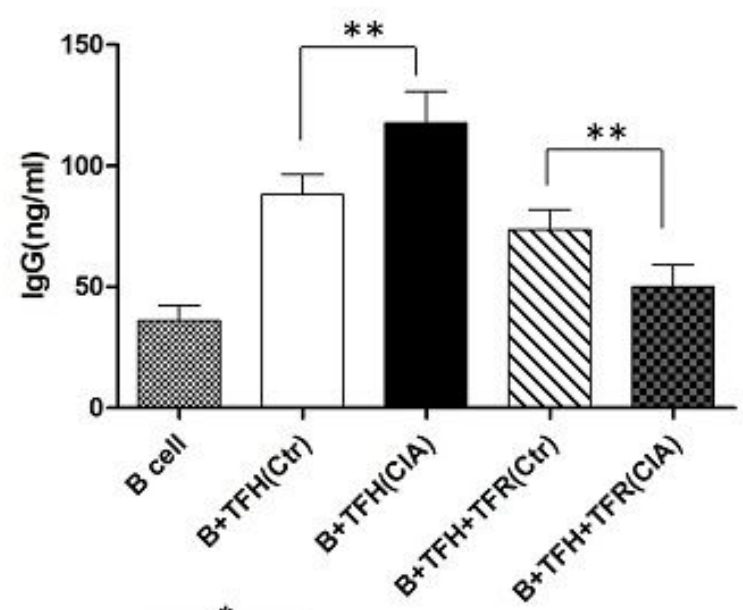

E

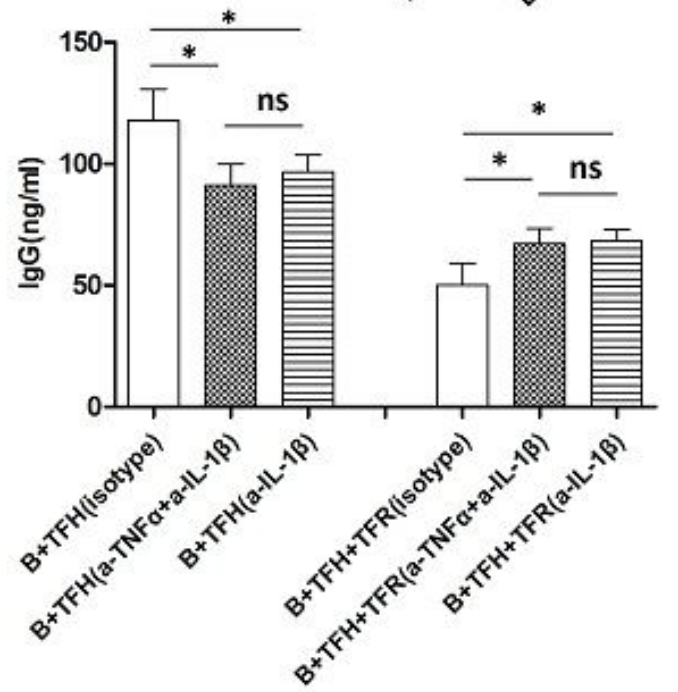

Figure 7

The function changes of TFH and TFR cells in CIA mice spleen by vitro culture. CD4+CXCR5+CD19-GITRTFH cells from 10-week time point CIA mice and control mice were co-cultured with CD19+ B cells (from spleen of CIA mice) for 5 days with anti-CD3 plus anti-IgM. For TFR cell cultures, CD4+CXCR5+CD19GITR+ TFR cells from two groups were added to the wells along with TFH and B cells (both from spleen of CIA mice). A. Representative flow cytometry dot plots of harvested cells showed GL 7+ germinal center $B$ cells that secrete $\lg \mathrm{G} 1$, and the numbers indicate $\mathrm{GL} 7+\lg \mathrm{G} 1+$ proportions in $\mathrm{CD} 19+$ cells. $\mathrm{B}$. Compare 
the percentage of $\mathrm{GL} 7+\lg G 1+$ cells in different culture systems. Each group included cells from spleen of four mice, and each sample was tested once. The result was expressed as mean with SD. C. Compare the concentrations of IgG in the supernatant of different cultures and each group included cells from four mice spleens, and each sample was tested twice. The result was expressed as mean with SEM. D\&E. TFH and TFR cells were derived from CIA mice injected with anti-inflammatory antibodies (anti-IL-1 $\beta$ or antiTNFa+anti-IL-1 $\beta$ ) or isotype IgG, and their functions were measured by co-cultures. GL $7+\lg G 1+$ cells and IgG levels in supernatant were measured and compared. Each group included cells from four mice spleens. ${ }^{* \star} p<0.001 ; * \star p<0.01 ; * p<0.05$; ns, not significant. 